\title{
Application of a Discrete Event Simulator for Healthcare Processes
}

\author{
Gregory Casier $^{1}$, Koen Casier $^{1}$, Jan Van Ooteghem ${ }^{1}$ and Sofie Verbrugge ${ }^{1}$ \\ ${ }^{1}$ Internet Based Communication Networks and Services research group IBCN, Ghent University, Belgium \\ Gregory.Casier@intec.ugent.be,Koen.Casier@intec.ugent.be,Jan.Vanooteghem@intec.ugent.be, \\ Sofie.Verbrugge@intec.ugent.be
}

Keywords: Discrete Event Simulator, eHealth, Process Simulation, KPI, Multi-objective optimization

\begin{abstract}
Hospitals are currently catching up with other industries to utilize IT tools for optimizing their patient, data and supply flows. The complexity of the processes and the amount of different resources and specialized personnel make a good view on the costs and effects of changes hard to understand. In light of this growing interest in healthcare towards leaner processes and better performance analytics, a process simulator was being developed. A Discrete Event Simulator (DES) allows the monitoring of Key Performance Indicators (KPIs) over existing processes and reveals opportunities for optimization. Opportunities to improve existing processes can easily be tested to verify possible gains before an actual implementation. Harmful side effects caused by changing the existing process can thus be measured on beforehand. The context in which the DES was being developed and an overview of the tool by explaining its different phases is provided, as well as an indication on potential further research topics and next developments.
\end{abstract}

\section{INTRODUCTION}

Hospitals consist of a complex set of clinical, material and information flows, which must come together in a point-of-care where the patient and the healthcare professional interact. These patient care processes are complex in nature (Mans et al., 2009) and often very isolated over the different disciplines that are present in a hospital (Lenz et al., 2002). While lean methods have been applied thoroughly in other sectors like car manufacturing, hospitals are lagging behind. The healthcare sector is currently in a lean learning phase to reduce inefficient operations and increase quality of service, but the synchronization of the different flows in an efficient manner is still a huge challenge. (Kim et al., 2006) A more patient oriented workflow, at the same time enabling efficient hospital operation, calls for innovative IT systems.

Several new methodologies are required to support this. One clearly identifiable need is to develop novel solutions for identifying, installing and monitoring an appropriate set of KPIs over the current processes. Process modeling methodologies will allow studying and optimizing process flows. By adding resource consumption information we can simulate, analyze and evaluate the impact of the proposed process optimizations for the chosen operational indicators (e.g. patient safety, quality, cost efficiency). Sim- ulating the proposal before implementing actual processes can avoid a lot of unnecessary costs, especially regarding the complex nature of processes in healthcare. (Bohmer, 2009)

For these reasons, a full discrete event simulator was constructed, capable of simulating a process based on statistical information on patient numbers and inter-arrival time, task timing, choices, etc. The simulator runs as realistically as possible a set of events through the process in which thousands of tasks can be simulated in less than a minute, and gather information on personal, patient and equipment timesheets (when in use or in treatment), waiting times and queue lengths, probabilities of events, etc.

These simulations allow us to monitor predefined KPIs and as such identify opportunities to improve the actual processes. Defining and adopting KPIs in a healthcare organization has proven useful by not only allowing the introduction of modern management approaches, but also by helping to revise the strategy. (Grigoroudis et al., 2012) Consequently, changes that might result in redesigned processes can also be checked on possible side effects before implementation.

Literature indicates different usage possibilities of simulation-based tools in a hospital environment. In (Jacobson et al., 2006) an overview is given of such implementations. A distinction is made between pa- 
tient flow optimization and analysis and health care asset allocation. Within the patient flow optimization there are several subtopics in which a DES has been applied. These include Outpatient Scheduling, Inpatient Scheduling and Admissions, Emergency Room Simulation Models, Specialist Clinics and Physician and Health Care Staff Scheduling. Also in (Günal and Pidd, 2010) a literature overview of discrete event simulators being applied in healthcare is being provided. The author indicates that the survey demonstrates the specificity of the studies. The simulators built have been found to be mostly unit or facility specific.

The remainder of this paper is organized as follows: first an overview of the iMinds eHealth project HIPS in which context the simulator was constructed is being provided. (HIPS, 2014) Secondly an overview of the DES is given and its different phases are being explained in more detail. Finally, we provide the reader with a general conclusion and make recommendations on further possibilities to extend the provided approach.

\section{HIPS PROJECT}

In this section a short overview of the project in which this simulator was developed is being provided. The HIPS project is an ongoing iMinds research project with both academic and industrial stakeholders in which the synchronization and optimization of different hospital flows is a key goal.

\subsection{Context}

According to the analytics at Gartner (Shaffer, 2012), "Healthcare is catching up with other industries in its demand for more timely and robust performance analytics and dashboarding". Many of the technological aspects are feasible today. But it has yet to become a reality because the healthcare supply chain, from manufacturer to patient, remains fragmented, with limited visibility and interconnection (Ebel et al., 2012).

Within a hospital environment a huge variety of processes and resources can be identified (Figure 1). These processes vary in nature. Processes can often not be planned in advance, and may occur in sequential or iterative mode (Bohmer, 2009). Most importantly, not being able to execute a process in a timely way may have a life threatening impact on the patient whereby the liability of the staff is very high. This makes a hospital environment an extremely complex environment to manage, unlike manufacturing organizations.

The mere size of the hospital has only a minor impact on the variety of processes. It is the number of supported treatments and specializations within a single hospital environment that has a high impact on this diverseness.

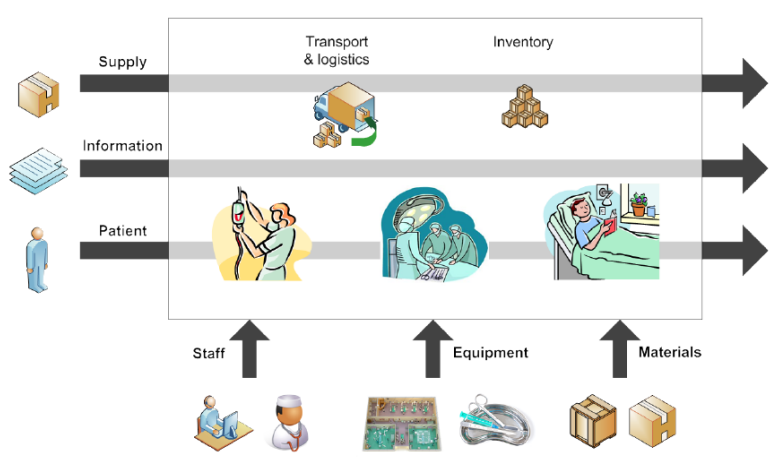

Figure 1: Overview of processes involved (AS IS)

Within this large variety of processes 2 main types of processes can be identified:

- The actual Treatment processes aiming to care, cure or help the patient, whereby treatment includes all technical medical and clinical activities related to diagnosis and therapy of illnesses, surgery, palliative care or other care; in a hospitalization mode or in a day treatment mode. This is the fundamental production process and determines how the care is given, and is reflected in a care path.

- The Supporting processes that target to serve or to support the treatment of a patient. Supporting processes basically include all non-treatment processes; finance, logistics, human resources, purchasing, etc.

One of the primary goals of the HIPS project is to design a methodology in order to optimize the supporting processes and align them with the treatment processes of the patient. This results with a single, integrated flow as depicted in Figure 2.

\subsection{Discrete Event Simulator within HIPS}

There are several reasons for which a DES is the appropriate tool for analyzing and optimizing processes within a hospital context.

1. A first reason is that the simulator allows the testing of different alternatives next to each other for a broad range of performances based on key performance indicators. 


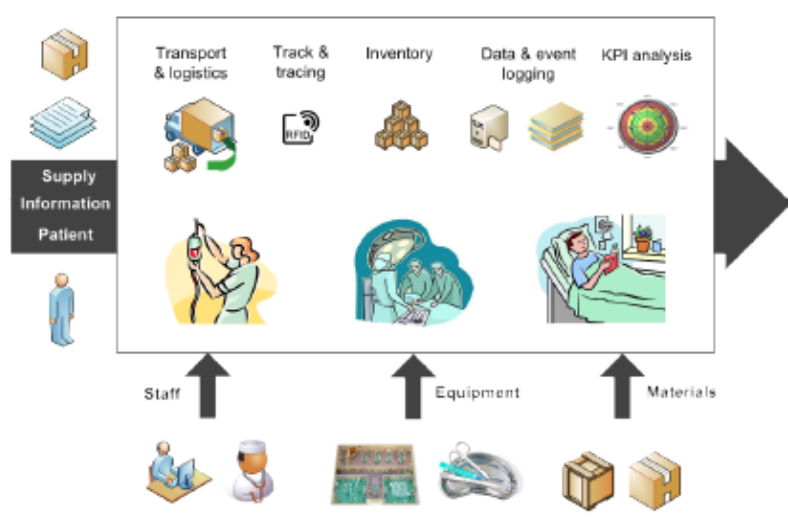

Figure 2: Overview of processes involved (TO BE)

2. Secondly, operational modeling is a trend of the last years and more and more operational processes are decently modeled and documented. Simulating these operational processes is the next step in discovering and visualizing problems such as bottlenecks, deadlocks, inefficient use of material and personal, increasing delays, etc.

3. A third reason we identify is that operational optimization needs to be run while closely monitoring the performance of these operational processes. The performance of an operational process, especially in healthcare, is not simply equal to the cost of executing the process. As such different KPIs of the operational process should be kept in mind while running an optimization, and keeping several such KPIs in mind at the same time is possible by using an operational simulation tool.

For these reasons, a discrete event simulator was developed and applied within the HIPS project. The simulator itself is being explained in the next paragraph.

\section{DISCRETE EVENT SIMULATOR}

\subsection{Motivation}

The key need that the simulator fulfills are summarized below.

1. Finding key performance indicators of the process

2. Finding opportunities for optimization

3. Checking for side effects of an optimization proposal

The identification and monitoring of KPIs over several simulations of an existing process allows us to identify opportunities to optimize this process even further. These opportunities can than also be virtually incorporated in the process and new simulations can provide insight in possible side effects.

Not only the motivation for applying a DES in a healthcare environment is of importance here, but also the architectural choice needs some clarification. There are plenty of DES software tools available, an clear overview was given in (Demyttenaere, 2014). From this list, the decision was made to implement a DES based on the MASON library (Multi-Agent Simulator Of Neighborhoods). There are several reasons for which this approach was chosen:

- A first major requirement was to narrow the list down by removing all commercial software packages. We wanted to have complete control over the code to have the freedom to expand it any way we deem necessary.

- Another requirement that guided our decision process was the preference to make use of the Activiti BPM (Business Process Management) platform (Rademakers, 2012). This requirement implies that the preferred language of the library/framework that will be selected is Java.

Due to these requirements the initial list was heavily reduced. From the remaining possibilities for a DES, the MASON library was selected, which will be further explained in the Simulation paragraph.

\subsection{Simulator Overview}

An overview of a simulated process can be found in Figure 3. Four different stages have been identified in the flow of simulator usage. These are being explained more into detail in the following paragraphs.
1. Input phase
2. Simulation phase
3. Output phase
4. Analysis phase

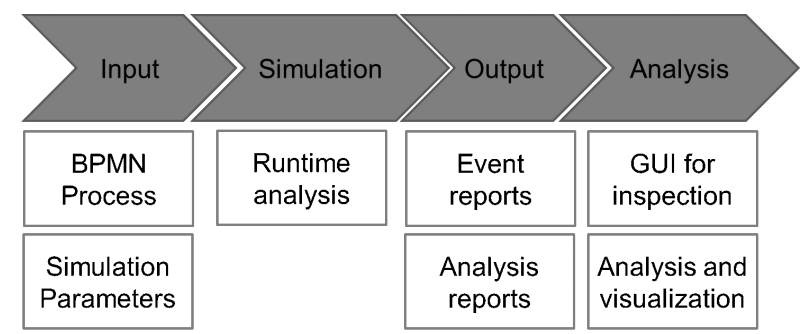

Figure 3: Overview of Simulator Steps 


\section{INPUT}

In a first stage, both the process we want to simulate as well as the parameters that define this process are being delivered as input. A visual representation of possible inputs is given in Figure 4

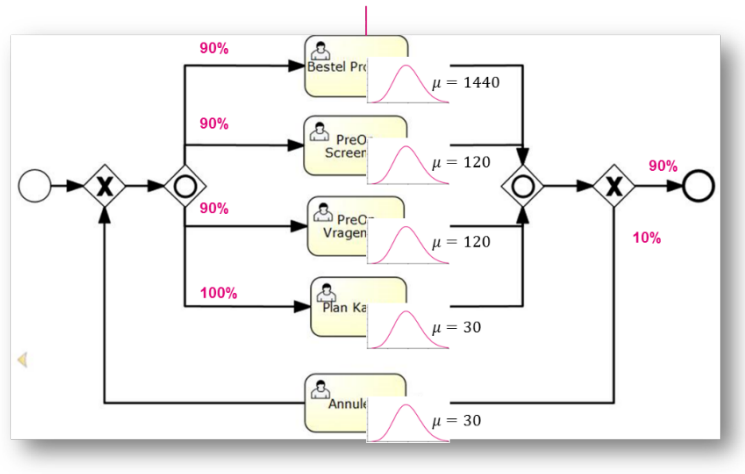

Figure 4: Input Phase

\subsection{Process Flow}

The process to be simulated has to be provided in the Business Process Model and Notation (BPMN) standard. BMPN is a well-known and widely acknowledged process flow standard with serialization in XML (Extensible Markup Language) format either in a proprietary or XPDL (XML Process Definition Language) format. Our tool works on both XPDL and proprietary Activity serialization format. Drawing the process outline in BPMN, serializing this scheme to $\mathrm{xml}$ and loading the $\mathrm{xml}$ in the simulator form the first steps in this process.

\subsection{Process Parameters}

Now that we have made the process outline known to the simulator, we also have to provide it with the context in which this process takes place. These context parameters are being provided in an xml file as well. Most of the Simulation Parameters are typically concerning costs and availability of resources and timings and probabilities of the different process steps (Tasks) involved.

Several examples of relevant process parameters in a hospital environmnent are provided below. A first category of parameters are those concerning the resources (employees, rooms, equipment, etc.)

- Concerning Employees (Nurses, Doctors, Surgeons, etc.)

- Availability (expressed in several blocks during a day)
- Cost (both fixed and variable)

- Resource Pool (for example, the group nurses can contain doctors, but not vice versa)

- Concerning Other Resources (Operating room, Examination room, etc. )

- Availability (expressed in several blocks during a day)

- Cost (both fixed and variable)

- Grouping (for example an Operating room could be used as an Examination room but not vice versa)

A second category of parameters for the process are those related to the execution of tasks in the process.

- Mean Time of task duration

- Distribution of task duration

- Resources required for a task

\section{SIMULATION}

In the next stage in the simulation process, the input from the previous step is being used to run the actual simulation. This is done by means of a Discrete Event Simulator, which models the operation of a system as a discrete sequence of events in time. "Discrete event simulation, based on a library of event models, is a way of simulating or characterizing cause-effect events that can be described as occurring at one particular moment in time a discrete event. These events are not continuous and have finite result outputs that are selected from a group of available outputs or calculated based on the inputs." (Hoare et al., 2002) During the simulation, convergence parameters are being monitored to check possible stop or convergence conditions.

The simulator was written entirely in the Java language and was built on top of several libraries, among which MASON. MASON stands for MultiAgent Simulator Of Neighborhoods and contains a fast discrete-event multi agent simulation library core. (Luke et al., 2004)

Several parameters are being monitored during the simulation to make sure the simulation gets halted when reaching convergence. The predefined criteria that make the simulator to stop running are listed below:

- Convergence of Full Process (Total duration of the process defined)

- Convergence of all Statistics (Every task's duration) 
- Maximum amount of Reports in which the data is stored is being reached

\section{OUTPUT}

When the simulation has ended for one of the reasons above, all detailed descriptions of the results are being outputted in xml-files. These include the steps that have been taken, the timings, the amount of resources utilized over time, etc.

All of the raw data is being kept in stand-alone $\mathrm{xml}$ files that are used to perform analyses on top of it. This allows us to perform different kind of analyses afterwards, without the necessity to rerun the simulation itself.

\section{ANALYSIS}

To be able to gain insights from these raw data files, there is a final Analysis phase. The custom GUI allows to open and visualize the data graphically. Predefined KPIs are being calculated from the output and these are being plotted in several graphs.

In the hospital context it is very likely that multiple, conflicting KPIs get defined. An overview of several of some of the KPIs we ran into during the HIPS project are the following (De Pourcq et al., 2015):

1. Patient related KPIs

(a) Number of new patient records, Number of finished patient records, etc.

(b) Mean lead time, maximum lead time, etc.

2. Process related KPIs

(a) Execution cost of the process, cost per patient, etc.

(b) Idle Time

3. Recourse related KPIs

(a) Usage of each resource (on average, minimal, etc.)

(b) Idle time of each resource

\section{Bottlenecks encountered}

(a) Tasks that cause bottlenecks

(b) Resources that cause bottlenecks

An optimal level for all of the KPIs is because of the conflicting nature impossible to achieve. Therefore there exist multiple equally rewarding possibilities and the most desirable solution is very much dependent on the preferences of the hospital governance. A multi-KPI overview of each process under consideration can be gained from the simulator and matching these values with the preferences might result in an optimal process design.

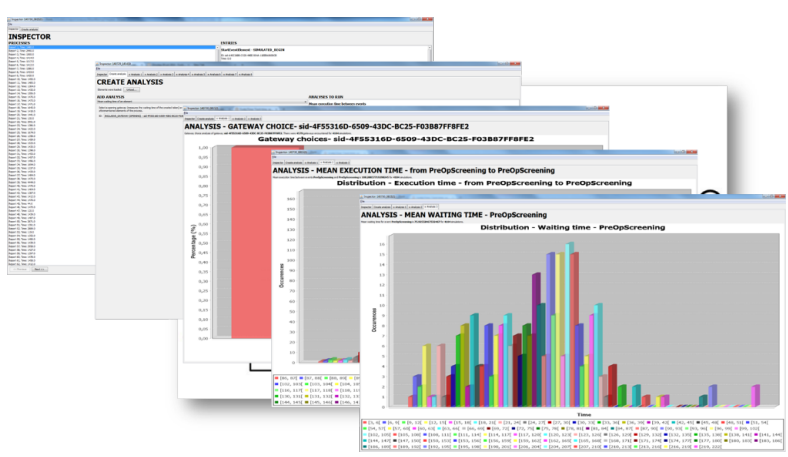

Figure 5: Analysis Phase

\section{NEXT STEPS AND FUTURE WORK}

The current context in which the simulation was developed is the hospital context. Both within and outside of this context can further research possibilities be identified.

Within the hospital environment coexist complex flows of patients, data and supplies. These processes are often susceptible for further optimization. The current simulator allows a lot of this setting to be modeled, but not all of it. Expanding the software to incorporate scheduling algorithms for example might be a valuable next step. Modeling different stock policies might be another possibility. Current research is ongoing concerning the matching of the KPIs with the preferences of hospital governance and visualizing the results. This way, hospital governance can steer the process by adjusting preference parameters and monitor results in a visually attractive way. Another line of work in progress is applying more complex resource selection methods in the simulator. More specifically ontology-based resource allocation in the simulation should more realistic resource selection in future simulations.

Further research possibilities outside of the hospital environment are ubiquitous. For this reason the simulator was built as generically as possible, being able to read in any type of process and resources. This allows completely different processes to be simulated without the need for imminent adjustments. 


\section{CONCLUSION}

In this paper we presented the key concepts of a discrete event simulator that can be applied for process simulation within a hospital flow optimization context. An overview of the simulator was being provided and the main steps (Input of process flow and resources, Discrete Event Simulation, Output of raw data and Analysis and visualization) have been explained. Simulating current processes has several advantages. It allows us to monitor key performance indicators of a process, identify opportunities for process improvement and check for possible side effects of process optimization proposals.

\section{ACKNOWLEDGEMENTS}

This research was carried out as part of the iMinds Health HIPS project. This project is co-funded by iMinds Health, and Amaron, Aucxis, H.Essers, AZ Maria Middelares and AZ Nikolaas.

\section{REFERENCES}

Bohmer, R. M. (2009). Designing care: aligning the nature and management of health care. Harvard Business Press.

De Pourcq, K., Gemmel, P., and Trybou, J. (2015). Measuring process performance in hospitals. In Proceedings 22th International Conference of European Operations Management Association (EUROMA).

Demyttenaere, P. (2014). Techno-economische Evaluatie Van Ehealth-diensten Met Behulp Van Ontologiegebaseerde Operationele Procesoptimalisatie. Master's thesis, Universiteit Gent, Ghent, Belgium.

Ebel, T., George, K., Larsen, E., Neal, E., Shah, K., and Shi, D. (2012). Strength in unity : The promise of global standards in healthcare. Technical Report October, McKinsey.

Grigoroudis, E., Orfanoudaki, E., and Zopounidis, C. (2012). Strategic performance measurement in a healthcare organisation: A multiple criteria approach based on balanced scorecard. Omega, 40(1):104-119.

Günal, M. M. and Pidd, M. (2010). Discrete event simulation for performance modelling in health care: a review of the literature. Journal of Simulation, 4(1):4251.

HIPS (2014). Hips, innovation by coordinating and optimizing patient, material and information flows in hospitals.

Hoare, R., Ahn, J., and Graves, J. (2002). Discrete event simulator. US Patent App. 10/043,847.

Jacobson, S. H., Hall, S. N., and Swisher, J. R. (2006). Discrete-event simulation of health care systems. In Patient flow: Reducing delay in healthcare delivery, pages 211-252. Springer.

Kim, C. S., Spahlinger, D. A., Kin, J. M., and Billi, J. E. (2006). Lean health care: What can hospitals learn from a world-class automaker? Journal of Hospital Medicine, 1(3):191-199.

Lenz, R., Elstner, T., Siegele, H., and Kuhn, K. A. (2002). A practical approach to process support in health information systems. Journal of the American Medical Informatics Association, 9(6):571-585.

Luke, S., Cioffi-Revilla, C., Panait, L., and Sullivan, K. (2004). Mason: A new multi-agent simulation toolkit. In Proceedings of the 2004 swarmfest workshop, volume 8.

Mans, R., Schonenberg, M., Song, M., van der Aalst, W. M., and Bakker, P. J. (2009). Application of process mining in healthcare-a case study in a dutch hospital. In Biomedical Engineering Systems and Technologies, pages 425-438. Springer.

Rademakers, T. (2012). Activiti in Action: Executable business processes in BPMN 2.0. Manning Publications Co.

Shaffer, V. (2012). Hype Cycle for Healthcare Provider Applications and Systems. Technical Report july, Gartner. 